\title{
Papel del entorno escolar en la transición a conductas activas en niños de
}

primaria.

Role of the school environment in the transition to active behavior in primary school children.

\section{Melchor Moreno, Ma. Teresa ${ }^{1}$, Montaño Corona, José Guadalupe ${ }^{1}$, Caudillo Cisneros, Cipriana $^{1}$ y Álvarez Delgado, Karina Isabel ${ }^{1}$}

${ }^{1}$ Universidad de Guanajuato

\section{CORRESPONDENCIA}

Ma. Teresa Melchor Moreno:

tmelchor2017@gmail.com

Universidad de Guanajuato
CÓMO CITAR

Melchor, Ma. T., Montaño, J. G., Caudillo, C. \& Álvarez, K. I. (2019). Papel del entorno escolar en la transición a conductas activas en niños de primaria. Revista de Ciencias del Ejercicio, 14 (2), pp. 9-20. Recuperado de: revistafod.uanl.mx

\section{RESUMEN}

El objetivo de este trabajo fue evaluar el papel del entorno escolar en el estadio o la etapa hacia la adopción de una conducta activa en la población de niños de 10,11 y 12 años de la ciudad de León Guanajuato. Material y métodos: Participaron 93 niños, 39 Mujeres y 54 hombres con edad promedio de $11 \pm 0.7$ años, se entrevistaron para evaluar el nivel de actividad física, entorno escolar y actitud ante la actividad física. Los puntajes del entorno escolar se obtuvieron bajo tres criterios: estimulación, infraestructura y nivel socioeconómico y clasificaron al entorno escolar en medio ambiente, adverso, regular y aceptable. La actitud se midió utilizando el Modelo Transteórico. Se utilizó estadística correlativa, descriptiva e inferencial y análisis de residuos de particiones, $\alpha=0.05$ Resultados: $\mathrm{Se}$ observó una prevalencia de sedentarismo del $43 \%$. La actividad física escolar no alcanza los criterios de frecuencia e intensidad requeridos (Strong, 2005). El medio ambiente escolar que prevaleció fue el regular ( $58 \%$ de alumnos). En las niñas el medio ambiente adverso fue significativamente asociado con un estado de contemplación, $(p>0.05)$. La actitud que prevalece en los escolares, es la preparación para la acción, $(P>0.05)$. Conclusión: El papel del entorno escolar en la transición a conductas activas en niños de primaria evaluados es ilusorio ya que a pesar de la oportunidad que representa la estancia en la escuela. Esta no ha sido debidamente aprovechada. Afectando principalmente a las niñas de nivel socioeconómico bajo.

Palabras clave: Sedentarismo, actividad física, conducta, entono escolar, medio ambiente.

\section{ABSTRACT}

The objective of this work was to evaluate the role of the school environment in the stadium or stage towards the adoption of an active behavior in the population of children of 10, 11 and 12 years of the city of León Guanajuato. Material and methods: 93 children, 39 women and 54 men with an average age of $11 \pm 0.7$ years participated, were interviewed to assess the level of physical activity, school environment and attitude towards physical activity. The school environment scores were obtained under three criteria: stimulation, infrastructure and socioeconomic level and classified the school environment in environment, adverse, regular and acceptable. The attitude was measured using the Transtheoretical Model. Correlative, descriptive and statistic inferential and partition residue analysis were used, $\alpha=$ 0.05. Results: A prevalence of sedentary lifestyle of $43 \%$ was observed. School physical activity does not meet the criteria of frequency and intensity required (Strong, 2005). The school environment that prevailed was the regular one (58\% of students). In girls, the adverse environment was significantly associated with a state of contemplation, $(p>0.05)$. The attitude that preponderate in the students is the preparation for the action, $(P>0.05)$. Conclusion: The role of the school environment in the transition to active behavior in primary school children evaluated is illusory because despite the opportunity that represents the stay in school. This has not been properly exploited. Affecting mainly girls of low socioeconomic status.

Key words: sedentary, physical activity, behavior, school environment, environment. 
Un estilo de vida activo es el que ha caracterizado al hombre desde sus orígenes, mantenía patrones de actividad física durante la caza, recolección y búsqueda de alimentos, actividades con alto grado de exigencia física. Hoy en día los trabajos son poco exigentes en materia de gasto calórico y los humanos se han vuelto cada vez más sedentarios (Archer \& Blair, 2011).

Las consecuencias del estilo de vida sedentario sobre el estado de salud del ser humano fueron ignoradas en los últimos 2000 años. Cuando Hipócrates dio origen a la epidemiología de la actividad física, varios epidemiólogos tales como: Galeno, Paracelso en la edad media, Bernardini, Ramazzini, durante la época de la Ilustración y William Heberden a mediados del siglo XIX, hasta Morris en 1994 entre otros, han demostrado los efectos de protección que la actividad física tiene contra enfermedades crónicas, evitando la mortalidad prematura. Investigaciones sustentan la premisa de que la actividad física, el entrenamiento con ejercicios y las mejoras en la capacidad cardiorrespiratoria son elementos esenciales en la prevención y el tratamiento de las enfermedades cardiovasculares inducidas por un entorno en el que la supervivencia ya no obliga al esfuerzo físico (Pafferbarger, Blair, \& Lee, 2001).

A pesar de todo es poca la población activa alrededor del mundo, proliferando día con día las conductas sedentarias desde épocas tempranas de la vida.

En un esfuerzo por dar respuesta a esta conducta de inactividad de la población se han llevado a cabo investigaciones para establecer que es lo que determina en los individuos realicen actividad física, Dishman, Sallis, \& Orestein (1985) después de una revisión minuciosa concluyeron que la participación regular en actividades físicas debe ser vista como un proceso dinámico en el que el mantenimiento y su adopción como estilo de vida son los resultados clave. En dónde; intención, capacidades personales, habilidades y conductas, compromiso y refuerzos emergen para que distintos grupos de población adopten finalmente estilos de vida activos saludables.

Para ayudar a lograr los objetivos de salud pública relacionados con la actividad física, las estrategias de modificación de estilos de vida y comportamientos individuales están orientadas a cambiar los entornos físicos, los que con más frecuencia se desarrollan, porque pueden beneficiar a todas las personas expuestas al medio ambiente (Brownson, Baker, Housemann, Brennan, \& Bacak, 2001).

Prochaska (1997) demostró que la transformación de la conducta no es solo un evento, sino un proceso dinámico que se cubre a través de etapas secuenciales como lo plantea en su modelo Transteórico.

El Modelo Transteórico (MT) del cambio del comportamiento en salud, propuesto por Prochaska (1979), y modificado por el mismo autor hasta llegar a la versión propuesta en 1992 ofrece posibilidades para planear y ejecutar intervenciones a partir de las características específicas de las poblaciones a quienes están dirigidas las acciones, y en quienes se detecta alguna de las cinco etapas o estados definidos: pre contemplación, contemplación, preparación, acción y mantenimiento para la adopción de conductas deseadas (Prochaska \& Wayne, 1997) 
En estos estados de cabio están involucrados cinco procesos básicos a diferente nivel:

La concientización a través de la retroalimentación, la catarsis con experiencias emocionales hacia la adopción de una conducta activa en este caso, elección de comportamiento a través de la auto determinación, estímulos condicionantes, control de contingencias.

El cambio de conducta se consigue a través de un avance progresivo de los estados de cambio o etapas desde la pre contemplación, a la contemplación, la preparación para la acción y la acción, y finalmente al estadio de mantenimiento, de esta forma se da el proceso de cambio, y cada una de estos está altamente relacionado con cada uno de los estados en los que se evalúa y clasifica a la persona (Prochaska \& Norcross, 1999).

Surge entonces la necesidad de indagar acerca de la influencia, que el medio ambiente escolar tiene sobre la conducta de los escolares ante la actividad física, e identificar sus características individuales y ambientales que delimitan o predisponen el proceso del cambio para intervenciones eficaces. El objetivo de este trabajo fue evaluar el papel del entorno escolar en el estadio o la etapa hacia la adopción de una conducta activa en la población de niños de 10,11 y 12 años de la ciudad de León Guanajuato.

\section{Material y Métodos}

Se evaluaron 93 niños (39 mujeres y 54 hombres) con una edad de $11 \pm 0.7$ años, obtenida por muestreo por conveniencia de acuerdo con accesibilidad a las escuelas y autorización de los padres para realizarla, los niños pertenecieron a cuatro escuelas representativas en las cuales se encontraron al menos 2 de los cuatro estratos sociales; clase baja, clase media baja, clase media, clase media alta, de acuerdo con la clasificación de niveles socioeconómicos en México descritos por Cheltenham \& Elgar (2004). Debido a que se observaron hasta tres niveles por escuela, la limitación de estas y manejo de datos se dispuso a clasificarlas en solo dos niveles socioeconómicos; el alto y el bajo de acuerdo a las cuotas escolares promedio que debían pagar, estan por encima o por debajo de $18,550.00 \pm 2,800.00$ anuales, costos reportados por un sondeo de colegios ubicados en diferentes zonas de la ciudad de León Guanajuato.

Para establecer el papel del entorno escolar en la actitud del niño ante la actividad física y nivel de actividad física se aplicaron 3 cuestionarios previamente validados para evaluar el entorno escolar en tres aspectos: Infraestructura, Currículo y docente, Nivel de actividad Física. También se entrevistó a los directores, maestros de grupo y de educación física para corroborar las horas curriculares, la promoción de la actividad física como activadores físicos.

Establecimiento del nivel de actividad física

El Nivel de Actividad Física (NAF) se obtuvo a través del cuestionario de actividad física modificado (CAF), validado para mexicanos adultos (López, Reyes, Castillo, Dávalos, \& González, 2001). Este cuestionario fue desarrollado, modificado y validado para niños mexicanos (Melchor, Montaño, Tovar, Díaz, \& Olivares, 2009). 
Se consideró a los niños con un estilo de vida sedentario quienes no realizaron actividad física de al menos 30 minutos por día a una intensidad moderada a intensa es decir aquellos quienes realizaban actividades con un gasto de energía menor a 3 METs, considerados como mínimos para alcanzar un beneficio para la salud (Strong, y otros, 2005).

\section{Actitud del niño ante la actividad física}

La conducta en relación con la actitud y el apego de la actividad física en la vida cotidiana de los escolares se midió utilizando el Modelo Transteórico de Prochaska \& Wayne (1997).

Para la determinación del estado de cambio en el que se encuentra la pobla ción escolar se desarrolló un cuestionario basado en el manual de consejería "Vida Sana" de Cecilia Albala, adaptado al tema de actividad física para niños de 10 a 12 años, que se aplica previo a un tratamiento de actitudes no sanas.

Las respuestas de cada uno de los niños permitieron clasificarlo en algún estadio o etapa del Modelo Transteórico: "Pre contemplación", "Contemplación", "Preparación para la acción" y "Acción" (Albala, Olivares, Sánchez, Bustos, \& Moreno , 2008).

Características que se evaluaron en el cuestionario de actitud con el modelo transteórico del comportamiento en la salud de Prochaska. Tabla 1.

Tabla 1.

Características que se evaluaron en el cuestionario de actitud en el MTP del comportamiento en la salud de Prochaska. Adaptadas del manual de Albala (2008).

\begin{tabular}{|c|c|}
\hline Estado de cambio & $\begin{array}{c}\text { Categorías en cada uno de los } \\
\text { estados de cambio }\end{array}$ \\
\hline Pre contemplación & $\begin{array}{l}\text { _Inconsciencia } \\
\text { _Desinterés } \\
\text { _Ninguna práctica deportiva o física } \\
\text { _ Indisposición }\end{array}$ \\
\hline Contemplación & $\begin{array}{l}\text { _Inconsciencia } \\
\text { _Ninguna práctica deportiva } \\
\text { _ Indisposición } \\
\text { _ Interés }\end{array}$ \\
\hline Preparación para la acción & $\begin{array}{l}\text { _Prevalece Inconsciencia } \\
\text { _Alguna práctica deportiva } \\
\text { esporádicamente } \\
\text { _ Disposición } \\
\text { _ Interés }\end{array}$ \\
\hline Acción & $\begin{array}{l}\text { _Consciencia } \\
\text { _ Práctica deportiva } \\
\text { _ Disposición } \\
\text { _ Interés }\end{array}$ \\
\hline
\end{tabular}


Evaluación del medio ambiente en el entorno escolar. El entorno escolar se evaluó con un cuestionario validado para reproducibilidad y fiabilidad con una $(p<.05)$. El cuestionario fue validado previamente para este propósito con una sub-muestra de 26 niños constituida de 6 niños elegidos al azar de cada una de las escuelas que confirmaron su participación, los niños contestaron el cuestionario en dos ocasiones con un periodo de dos semanas entre la aplicación uno y dos. Se utilizó correlación intercalase para confiabilidad y t-studen para reproducibilidad y fue sometido al método de consistencia interna alfa de Cronbach. Esta encuesta evaluó el entorno tomando en consideración la estimulación y motivación del docente; promoción de actividades físicas y deportivas, horas de educación física programadas curricularmente, infraestructura; aéreas, espacios en la escuela para la práctica deportiva y activación y nivel socioeconómico.

El entorno fue evaluado con un puntaje Likert de 0 a 2, donde " 0 " corresponde a un puntaje mínimo aportado por ese entorno de 0 al $25 \%$ " 1 " corresponde a un puntaje aportado, del 26 al $75 \%$ y "2" corresponde al de $76 \%$ al $100 \%$. Posteriormente de acuerdo con el puntaje se determinó si el medio ambiente escolar se consideraba un medio ambiente "adverso", "regular" o "aceptable", para posteriormente estimar el grado de influencia con la que cada uno de ellos contribuye sobre el nivel de actividad física, y estado de cambio de conducta de los escolares.

\section{Análisis Estadístico}

El tamaño de la muestra relativa a la población se determinó de acuerdo con la prevalencia de jóvenes que permanecen sentados frente al monitor por más tiempo que el recomendado por OMS, reportada por adolescentes en una investigación realizada en la localidad por Montaño (2007) a un nivel de confianza de $95 \%$, con un error del 0.05 , por bloques de edad y género, con un universo de 176 hombres y 182 mujeres.

Se utilizó estadística correlativa intercalase,t-student para validación y confiabilidad de cuestionarios y Alfa de Cronbach, para fiabilidad de la consistencia interna del instrumento. También se utilizó estadística descriptiva e inferencial, y la prueba Ji cuadrada para k muestras o grupos independientes y análisis de residuos de particiones para determinar la contribución de variables sobre una condición específica, a un nivel de confiabilidad para una distribución normal Bidireccional $\alpha=0.05$. Específicamente para determinar el medio ambiente ("aceptable", "regular" o "adverso") que tiene mayor influencia sobre la transición hacia un estilo de vida activo.

\section{Consideraciones éticas}

Toda la investigación se realizó siguiendo las recomendaciones de la declaración de Helsinki para la investigación biomédica de seres humano (Asociacion Médica Mundial, 2008). Solo se evaluaron a menores quien previó a la evaluación sus padres o tutores legales autorizaron y firmaron la carta de consentimiento informado bajo pleno conocimiento de que estarían participando en una investigación, en los protocolos y características de las evaluaciones y validación de cuestionarios, el derecho de retirarse de la evaluación en cualquier momento que ellos lo decidan, y/o lo consideren riesgoso. A cada uno de los padres se les explicó que para la publicación de los datos se garantizaba la confidencialidad de 
los datos y la información que se generara durante las entrevistas, además se les indicó que podían estar presentes en las evaluaciones o nombrar a un comité de padres que las presenciara, y que en todo momento ellos tendrían la oportunidad hacer preguntas con el propósito de disipar toda duda.

\section{Resultados}

Prevalencia de sedentarismo y nivel de actividad física en la muestra de escolares. En lo que respecta al sedentarismo los niños quienes realizan actividades durante el día por debajo de 3 METs. En la Tabla 2 se muestra la prevalencia de sedentarismo en escolares.

Tabla 2

Prevalencia de sedentarismo en escolares muestra local.

\begin{tabular}{cccc}
\hline Estilo de vida & $\begin{array}{c}\text { Hombres } \\
(\%)\end{array}$ & $\begin{array}{c}\text { Mujeres } \\
(\%)\end{array}$ & $\begin{array}{c}\text { General } \\
(\%)\end{array}$ \\
\hline Sedentario & 37 & 51 & 43 \\
Activo & 63 & 49 & 57 \\
\hline Total & 100 & 100 & 100.0 \\
\hline
\end{tabular}

El papel del entorno escolar en la realización de la actividad física, de moderada a intensa de al menos 30 minutos/ día, fue nulo en todos los niveles socioeconómicos. Ya que las actividades que se realizan principalmente durante la clase educación física y recreo fueron clasificadas ligeras de acuerdo con López et al. (2001), las cuales consistieron básicamente en jugar volibol, basquetbol recreativo, fútbol y trotar en periodos de tiempo menores a $15 \mathrm{~min}$. (Strong, 2005).
Tabla 3.

Horas de actividad física reportadas por los escolares en 24 horas

\begin{tabular}{lcc}
\hline Actividades & Hombres & Mujeres \\
en 24 horas. & $\mathrm{X} \pm \mathrm{DE}$ & $\mathrm{X} \pm \mathrm{DE}$ \\
\hline Ligeras & $23.4 \pm 0.4$ & $23.6 \pm 0.3$ \\
Moderadas & $0.4 \pm 0.3$ & $0.2 \pm 0.26$ \\
Intensas & $0.2 \pm 0.2$ & $0.1 \pm 0.20$ \\
\hline
\end{tabular}

Es necesario puntualizar que los escolares que realizan ejercicio más de 30 minutos por día de alta intensidad son de nivel socioeconómico alto y lo realizan independientemente del entorno escolar.

Medio ambiente escolar. De acuerdo con la clasificación de medio ambiente en el entorno escolar se observó lo que a continuación se presenta en la Tabla 4.

El $67 \%$ de los escolares no cuentan con las condiciones ambientales aceptables para iniciar la transición hacia un cambio de conducta que los lleve a un estilo de vida más activo. La mayor parte de los escolares que están inmersos en un medio ambiente "aceptable" son del nivel socioeconómico alto $91 \%$ en comparación con los niños de los niveles bajos con sólo $1 \%$ ( $p>$.05). Los niños de nivel socioeconómico bajo se encuentran en su mayoría en un ambiente regular.

Tabla 4.

Medio ambiente escolar que prevalece

\begin{tabular}{lcccc}
\hline Ambiente & $\begin{array}{c}\text { Adverso } \\
\mathrm{n}(\%)\end{array}$ & $\begin{array}{c}\text { Regular } \\
\mathrm{n}(\%)\end{array}$ & $\begin{array}{c}\text { Aceptable } \\
\mathrm{n}(\%)\end{array}$ & Total \\
\hline Hombres & $7(13)$ & $36(67)$ & $11(20)$ & $54(58 \%)$ \\
Mujeres & $4(10)$ & $22(56)$ & $13(33)$ & $39(42 \%)$ \\
\hline Total & $11(12)$ & $58(55)^{\star}$ & $24(33)$ & $93(100)$ \\
\hline
\end{tabular}


En lo que respecta a las niñas inmersas en un medio ambiente "aceptable" el $85 \%$ son del nivel socioeconómico alto y el $15 \%$ corresponde al nivel socioeconómico bajo. La mayor parte de las niñas se encuentra en un medio ambiente "regular" y "aceptable". El medio ambiente adverso se presenta en el doble de las niñas de nivel socioeconómico bajo en Comparación con el nivel socioeconómico alto.

Actitud de los escolares ante la Actividad física y el papel del entorno escolar. En la Tabla 5 se pueden observar las etapas conductuales en que se encuentran los niños.

Tabla 5.

Etapas conductuales de Prochaska en las que se encuentran los escolares

\begin{tabular}{lccc}
\hline Etapa o estadio & $\begin{array}{c}\text { Hombres } \\
\%\end{array}$ & $\begin{array}{c}\text { Mujeres } \\
\%\end{array}$ & $\begin{array}{c}\text { General } \\
\%\end{array}$ \\
\hline Pre contemplación & 0 & 1 & 1 \\
Contemplación & 2 & 3 & 5 \\
Preparación & 38 & 22 & 60 \\
Acción & 18 & 16 & 34 \\
\hline Total & 58 & 42 & 100 \\
\hline
\end{tabular}

La actitud mostrada en los escolares con respecto al medio ambiente observado en el entorno escolar nos muestra que en general un cambio positivo en la conducta más activa se manifiesta a partir de un entorno regular e influye para que los escolares alcancen el estadio de acción, Tabla 6 y Tabla 7.
Tabla 6.

Asociación entre el medio ambiente y etapas de actitud ante la actividad física de escolares mujeres



La diferencia porcentual en las mujeres sugiere que la actitud en "pre-contemplación" fue influenciada básicamente por el medio ambiente escolar adverso, $(p>.05)$. Por otro lado también podemos observar que el medio ambiente que mayormente influencio en las niñas con una mejor actitud ante la actividad física en los estadios de "pre-acción" y "acción" fue el medio ambiente regular, ya que las diferencias porcentuales fueron de 7 a 1 y de 2 a 1 con respecto al medio adverso (33.3\% $+17.9 \% / 5.1 \%+2.6 \%$ y con el medio ambiente aceptable $(33.3 \%+17.9 \%$ / $15.4 \%+15.4 \%)$, respectivamente. Aunque los valores residuales individuales indicaron que existió una influencia del medio ambiente regular sobre el estadio o etapa de "pre-acción" (0.7) y una influencia del medio ambiente aceptable con el estadio o etapa de "acción". Estos valores no indicaron que la relación entre las variables fuera significativa a un intervalo de confianza del $95 \%$, y se acepta una relación de independencia entre estas las variables. 
Con respecto a los niños en la Tabla 7, se observa que los niños no presentan estado de pre contemplación.

Tabla 7.

Asociación entre el medio ambiente y etapas de actitud ante la actividad física de escolares hombres

\begin{tabular}{|c|c|c|c|c|c|c|c|}
\hline & & \multicolumn{6}{|c|}{ Etapas de Actitud } \\
\hline & & & Pre-Con & Con & Pre- & & \\
\hline & & & Templa & Templa & Acción & Acción & Total \\
\hline & & Recuento & & $\mathrm{O}_{\mathrm{a}}$ & $44_{a}$ & $3 a$ & 7 \\
\hline & Adverso & $\%$ del total & - & $0.0 \%$ & $7.4 \%$ & $5.6 \%$ & $13.0 \%$ \\
\hline & & Residuo corregido & & -.6 & -.5 & .7 & \\
\hline Medio & & Recuento & & $2 a$ & $23_{\mathrm{a}}$ & $9_{\mathrm{a}}$ & 34 \\
\hline \multirow[t]{7}{*}{ Ambiente } & Regular & $\%$ del total & _ & $3.7 \%$ & $42.6 \%$ & $16.7 \%$ & $63.0 \%$ \\
\hline & & Residuo corregido & & 1.1 & .6 & -1.0 & \\
\hline & & Recuento & & $\mathrm{O}_{\mathrm{a}}$ & $8 \mathrm{a}$ & $5 a$ & 13 \\
\hline & Aceptable & $\%$ del total & _- & $0.0 \%$ & $14.8 \%$ & $9.3 \%$ & $24.1 \%$ \\
\hline & & Residuo corregido & & -.8 & -.3 & .6 & \\
\hline & Total & Recuento & & 2 & 35 & 17 & 54 \\
\hline & & $\%$ del total & - & $3.7 \%$ & $64.8 \%$ & $31.5 \%$ & $100.0 \%$ \\
\hline
\end{tabular}

En estos resultados podemos observar el mismo comportamiento de los niños que en las niñas La diferencia porcentual en los hombres sugiere que la actitud en "contemplación" no fue influenciada por el medio ambiente adverso sino por el medio ambiente regular.

Al igual que en la niñas el medio ambiente que fundamentalmente influenció para que los niños se encuentren en los últimos estadios hacia un cambio de actitud en "pre-acción" y "acción" fue el medio ambiente regular, ya que las diferencias porcentuales fueron de 4 a $1 \mathrm{y}$ de 2 a 1 con respecto al medio adverso $(42.6 .3 \%+16.7 \% / 7.4 \%+5.6 \%)$ y con el medio ambiente aceptable $(42.6 .3 \%+$ $16.7 \% / 14.8 \%+9.3 \%)$, respectivamente.

Los valores residuales individuales no indicaron que la relación entre las variables fuera significativa a un intervalo de confianza del $95 \%$, y se acepta una relación de independencia entre las variables.

En un análisis adicional se observa que un medio ambiente regular en las niñas, sin hacer diferencias socioeconómicas, evita que permanezcan en un nivel de pre contemplación y favorece más a que las mujeres lleguen alcanzar un nivel de acción que un nivel de pre acción, en especial si se trata de las niñas de niveles socioeconómicos bajos ya que las niñas con un nivel socioeconómico alto son significativamente influenciadas por un entorno aceptable más que por el medio ambiente regular dando la impresión de que este ambiente tiene alcances solo para un nivel de pre acción en los niveles socioeconómicos más altos $(p<.05)$.

Con respecto a los hombres al igual que en las mujeres, el medio ambiente que contribuyó significativamente en la disminución de niños en "acción", e influyó a su vez para que más hombres fueran clasificados en el estadío de contemplación fue el medio ambiente adverso. Sin embargo, se observa en los niños en general no haciendo diferencias socioeconómicas que solo un medio ambiente aceptable favorece a que ellos puedan acceder a un estado de acción y un ambiente regular es suficiente para llegar a la pre-acción.

En las mujeres de nivel socioeconómico bajo el entorno escolar es determinante en la conducta activa de las mujeres. Sin embargo, un medio ambiente escolar apenas regular, facilita una transición a las conductas esperadas

En el caso de los hombres de nivel socioeconómico alto, al igual que en las mujeres el entorno escolar tampoco es determinante para la transición a conductas activas. 
En los hombres de nivel socioeconómico bajo la barrera para trascender es un medio ambiente escolar adverso y un medio escolar regular.

Transición hacia un estilo de vida más activo. De acuerdo con las características que los escolares respondieron en el cuestionario de actitud de Prochaska. El estado de cambio de la población de escolares se distribuyó de la siguiente manera. EL $1 \%$ se encuentra en etapa pre contemplación, permanece inconsciente, apático ante la actividad física, no practica ninguna actividad física, siempre esta indispuesto, cabe señalar que todas son mujeres.

El $5 \%$ está en etapa de contemplación estos escolares reportaron ser inconscientes no practican ninguna actividad física siempre esta indispuesto a activarse, pero ya muestra algún interés en alguna actividad.

El $60 \%$ de ellos se encuentran en "preparación a la acción" aún persiste la inconsciencia, pero ya práctica alguna actividad física está dispuesta a activarse e incluso puede ser catalogado como activo al realizar más de 30 minutos de actividad física de moderada a intensa además muestra interés por esta.

En general los que ya han iniciado el proceso de transformación y se encuentran en el estadio de "acción", son $34 \%$ de los escolares, estos se caracterizan de acuerdo con sus respuestas por ser conscientes de los beneficios de la actividad física sobre su salud y experimentan el bienestar en su cuerpo al activarse físicamente y disfrutan al realizarla.
En otro apartado haciendo referencia al $57 \%$ de los niños considerados activos por realizar de 30 a 45 minutos de actividad física de intensa a moderada, sólo el $24 \%$ estos son los que realmente se encuentran en el estadio de "acción", clasificados así por sus respuestas en el cuestionario de actitud de Prochaska, es decir son conscientes de lo que representa la actividad física en su vida y en su salud, están dispuestos siempre activarse y son muy activos. Los 33\% restantes son activos de oportunidad, su medio ambiente se los permite, puede ser que de alguna forma perciben el bienestar que les provoca la activación, lo hacen por diversión, distracción o por sentirse acompañados. Sin embargo, son inconscientes, situación que puede estancarlos en el estado de cambio de la pre acción y nunca puede llegar a consolidarse un cambio en su estilo de vida.

Con respecto a las niñas sólo el $49 \%$ clasificada como activas por realizar actividades de 30 a 45 minutos diarias de moderadas a intensas, de estas el $30 \%$ están conscientes de los beneficios de la activación, les agrada, siempre están dispuestas a participar y practican alguna actividad física o deporte. Las niñas restantes el 19\% son activas que no pueden ser consideradas en el estadio de acción por sus respuestas en el cuestionario de actitud de Prochaska, al igual que los niños son activos de oportunidad.

\section{Discusión}

La Encuesta Nacional de Salud y Nutrición, ENSANUT (2012) reporta que un $58 \%$ de los adolescentes Mexicanos son sedentarios, del 2006 y 2012, existió un 
incremento de sedentarismo en niños de esta edad del 18\%. Aproximadamente un incremento de sedentarismo infantil del 3\% anual. A pesar de los resultados nacionales el $43 \%$ de nuestros escolares se clasificaron como sedentarios, porcentaje menor al de la media nacional, sin dejar de ser trascendental (Gutiérrez, y otros, 2012).

Investigaciones coinciden que las escuelas son los lugares con la mayor oportunidad para que los niños inicien a experimentar, disfrutar la actividad física y adopten estilos de vida sanos que trasciendan a su vida adulta. (Ennis, 2013; Huberty, Dinkel, Coleman, \& Beighle, 2012; Lowry, Wechsler, Kann, \& Collins, 2001; Chen, 2012). En este sentido en el 2008, en México a través de la Secretaría de Educación Pública se pone en marcha la política nacional de actividad física escolar, en donde el objetivo central fue que diariamente en todas las escuelas públicas y privadas de educación primaria se impartiera por parte de los maestros de educación física o maestros de grupo, sesiones diarias de 10 o 15 minutos hasta acumular 30 minutos.

Sin embargo a pesar de los programas y políticas que se establecen, con el fin de hacer de la actividad física un estilo de vida desde la escuela parecen no tener ningún efecto ya que de acuerdo con los resultados de esta investigación, estas actividades se han estado diluyendo, de acuerdo con la frecuencia de las actividades físicas o nulas actividades físicas que se realizan en la escuela y la intensidad de estas, que en su mayoría se catalogaron como ligeras de acuerdo con López et al. (2001), reportadas por alumnos y maestros.
El entorno escolar no es estadísticamente aceptable $(p>.05)$, el medio ambiente aceptable se observó en mayor medida en las escuelas de nivel alto y fueron clasificadas así por alcanzar un alto puntaje en el número de horas de educación física programadas por semana, actividades y torneos extracurriculares y por sus instalaciones e infraestructura deportiva a pesar del bajo puntaje obtenido por la actitud docente.

El medio ambiente regular fue el que prevaleció, 6 de cada 10 niños están inmersos en un ambiente regular ( $p>.05$ ). En el otro sentido el medio ambiente que influyó significativamente $(p>.05)$ para que las niñas, en específico las de nivel bajo permanecieran en el estadio de "Pre contemplación" fue el medio ambiente adverso, el cual fue clasificado así porque las niñas de $5^{\circ}$ y $6^{\circ}$ de las escuelas públicas no tienen clase de educación física, las instalaciones deportivas son mínimas y siempre están ocupadas con clases de grupos de niños más pequeños, nula estimulación y motivación por parte del docente.

Para algunas niñas de nivel socioeconómico bajo la escuela es la única oportunidad para la realización de las actividades y físicas por lo que es potencialmente difícil que estas niñas inicien un proceso de adopción de estilos de vida saludables y de prevención para su salud.

La respuesta de los niños evaluados en este trabajo muestra que el proceso de aprendizaje de conceptos, valores y actitud ante la actividad física existe. Sin embargo, este proceso se da fuera del entorno escolar a través de los padres, ya que una gran parte de los niños en acción reportan que alguno de sus padres practica alguna actividad física. 
Se ha demostrado por varios autores que la infraestructura adecuada en las escuelas correlaciona significativamente con la adopción de la actividad física como estilo de vida en los niños, por varios factores por la seguridad de dejar a los niños en la institución, porque no tienen que trasladarlos a las unidades, por el costo que involucra acudir a los centros y unidades deportivas y de recreación aun las públicas cuyos costos impiden que gran parte de los sectores bajo y medio tengan acceso a estas instalaciones (Haug, Thorsheim, Sallis, \& Samdal, 2010).

\section{Conclusiones}

Es claro que el papel del entorno escolar es indispensable para lograr que los escolares realicen la actividad física requerida para beneficio de la salud, principalmente en las niñas de nivel socioeconómico bajo, de acuerdo con los resultados obtenidos.

El $32 \%$ de los escolares se encuentran en la etapa o estadio de "acción", es decir, están conscientes de los beneficios de practicar actividad física y la realizan con regularidad.

A pesar de su entorno, un $89 \%$ de las niñas están en los últimos estadios hacia una actitud deseada.

El sedentarismo está presente en ambos niveles socioeconómicos, combinando niños y niñas con una ligera tendencia a un mayor sedentarismo en el nivel socioeconómico bajo.

De las niñas de nivel socioeconómico bajo sólo una quinta parte se clasificaron como activas.
Los escolares clasificados como activos complementaron los estándares fuera del entorno escolar y estos fueron en su mayoría los pertenecientes a escuelas privadas, ya que tanto en la escuela pública como en la privada no se están cumpliendo los estándares de frecuencia e intensidad recomendados para lograr un beneficio a la salud.

\section{Referencias}

Albala , C., Olivares, S., Sánchez, H., Bustos, N., \& Moreno, X. (2008). Manual de Consejeria en Vida Sana. Instituto de Nutrición y Tecnología de los Alimentos.

Archer, E., \& Blair, S. (2011). Physical activity and the prevention af cardiovascular sisease: from evolution to epidemiology. Progress in cardiovascular diseases, 53(6), 387-396.

Asociacion Médica Mundial. (2008). Declaración de Helsinki. Principios éticos para las investigaciones con seres humanos. 59a Asamblea. Seúl.

Brownson, R., Baker, E., Housemann, R., Brennan, L., \& Bacak, S. (2001). Enviroment and policy determinants of physical activiti in the United States. American Journal of Public Health, 91(12).

Chen, A. (2012). On Childhood obesitu prevention: "Exercise is medicine vs Exercise is vaccine". Journal of Sport and Health Science, 6(2), 14-31. doi:10.1016/j.jshs.2012.11.003

Dishman, R., Sallis, J., \& Orestein, D. (1985). The determinants of physical activity and exercise. Public Health Reports, 100(2), 158-171. 


\section{Referencias}

Ennis, C. (2013). Reimagining professional competencie in physical education. HHS Public Access, 19(4), 662-672.

Gutiérrez, J., Rivera, D., Shamah, T., ViIlarpando, S., Franco, A., Cuevas, L., Hernández, A. (2012). Encuesta Nacional de Salud y Nutrición. Resultados Nacionales. Instituto Nacional de Salud Pública.

Huberty, J., Dinkel, J., Coleman, A., \& Beighle, B. (2012). The role of school in childen's physical activitu participation: staff perceptions. Health Esucation Research, 27(6), 986-995.

López, J., Reyes, S., Castillo, L., Dávalos, A., \& González, J. (2001). Reproducibility and sensitivity of a questionnaire on physical activity in a Mexican population. Salud Pública de México, 43(4), 306-12.

Lowry, R., Wechsler, H., Kann, L., \& CoIlins, J. (2001). Recent trends in participation in physical education among US high school students. The Journal of School Health, 71, 145-52.

Melchor, T., Montaño, J., Tovar, J., Díaz, F., \& Olivares, P. (2009). Actividad física, gasto calórico y otros índices de sobrepeso en adolescentes. Memorias 9. Reunión de Egresados de Ciencias Médicas, (págs. 38-42). León.

Pafferbarger, R., Blair , S., \& Lee , I. (2001). A history of physical activity, cardiovascular health and longevity: the scientific contribution of Jeremy N Morris, DSc, DPH, FRCP. International Journal of Epidemiology, 30(5), 1184-92.
Prochaska, J., \& Norcross, J. (1999). Systems of Psychotherapy: A Transtheoretical Analysis (Cuarta ed.). Brooks/Cole Publishing Company.

Prochaska, J., \& Wayne, F. (1997). The Transtheoretical Model of Health Behavior Change. American Journal of Health Promotion, 12(1), 38-48.

Secretaria de Educación Pública. (2008). Programa Nacional de Activación Física. Morelia, Michoacán: Gobierno Federal.

Secretaría de Salud Pública. (2002). Programa de atención a la salud de la adolescencia. México: Secretaría de Salud Pública.

Strong, W., Malina, R., Blimikie, J., Daniels, S., Gutin, S., \& Hergenroeder, A. (2005). Evidence based physical activity for school-age youth. The Journal of Pediatrics, 146(6), 732-737. 